\title{
Raman DFB Fiber Laser with Truly Unidirectional Output
}

\author{
Jindan Shi, Shaif-ul Alam, Morten Ibsen \\ Optoelectronics Research Centre, University of Southampton, Highfield, Southampton, SO17 1BJ, UK \\ jxs@orc.soton.ac.uk \\ Abstract: We report a single-frequency, kilohertz-linewidth $(<2.5 \mathrm{kHz}), 30 \mathrm{~cm}$-long Raman \\ distributed-feedback fiber laser with unidirectional output. The threshold power, slope \\ efficiency and maximum output power are observed to be $\sim 980 \mathrm{~mW}, 7.7 \%$ and $296 \mathrm{~mW}$ \\ OCIS codes: (060.3735) Fiber Bragg gratings; (140.3490) Lasers, distributed-feedback; (140.3550) Lasers, Raman.
} respectively.

\section{Introduction:}

Raman gain-based distributed-feedback (R-DFB) fiber lasers represents an attractive proposition for generating single-frequency, narrow linewidth and low noise laser light at wavelengths outside of the traditional bands covered by rare-earth (RE) doped fiber lasers [1-3]. The R-DFB fiber laser is based on a passive Raman fiber with a Bragg grating written directly into the fiber core. Typically a discrete $\pi$ phase-shift is required at or close to the center of the Bragg grating to ensure both low lasing threshold and single-frequency operation. In most cases fiber lasers ideally should be operating with single-side output to maximize the slope efficiency [4]. In previous demonstrations this has been achieved by using an asymmetric $\pi$ phase-shifted Bragg grating structure $[4,5]$. In this work, we present an R-DFB fiber laser with truly unidirectional output from a $30 \mathrm{~cm}$ long center $\pi$ phase-shifted DFB grating with a slightly tapered refractive index profile. We show that the laser operates in a single-frequency at $1117.71 \mathrm{~nm}$ with a linewidth of $<2.5 \mathrm{kHz}$ and an output power splitting ratio of $>60 \mathrm{~dB}$. The threshold pump power and the maximum output power were measured to be $\sim 980 \mathrm{~mW}$ and $\sim 300 \mathrm{~mW}$, respectively.

\section{Experimental setup}
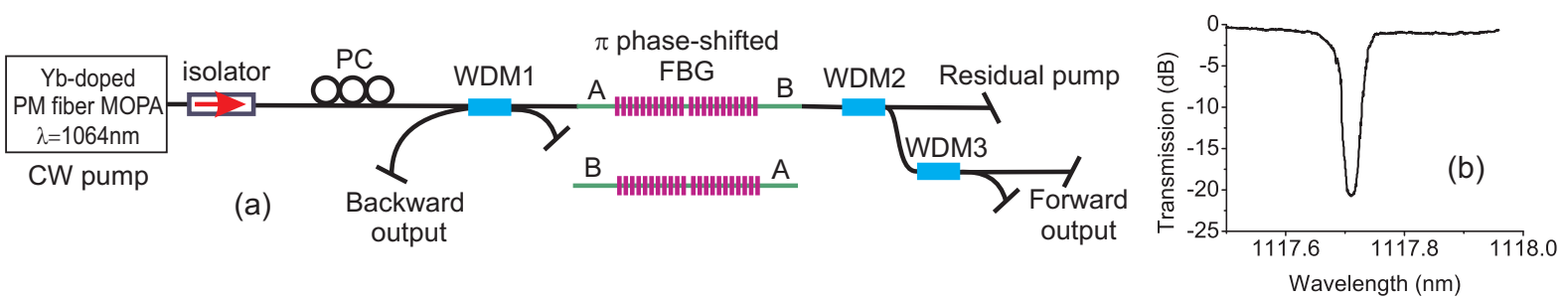

Fig. 1 (a) Schematic diagram of experimental setup; (b) DFB grating transmission spectrum with a resolution of $0.01 \mathrm{~nm}$.

The schematic diagram of the experimental setup is shown in Fig. 1(a). A continuous-wave (CW), linearly polarized, 1064nm Yb-doped PM fiber MOPA was used as the pump source. The output of the fiber MOPA was spliced to a high power isolator followed by a polarization controller (PC). The latter was used to align the polarization state of the pump to the DFB grating. $1064 \mathrm{~nm} / 1117 \mathrm{~nm}$ wavelength division multiplexers (WDM1, $2 \& 3$ ) were used at both backward and forward ends of the DFB grating, to separate the R-DFB signal from the pump. All fiber end-facets were angle-cleaved to prevent end-feedback. All WDMs and the DFB grating were mounted on heat sinks to effectively dissipate any potential heat generated from the laser and maintain good temperature uniformity of the sample. Note that all the passive components used in this experiment are non-PM.

A commercially available photosensitive silica fiber (PS980, Fibercore) closely matched in numerical aperture (NA) and core-diameter to Hi1060 was chosen as the Raman DFB fiber laser host to ensure a low splicing loss with standard components, and a 30cm-long, center $\pi$ phase-shifted DFB grating was written directly into the fiber using a $244 \mathrm{~nm}$ CW UV-laser. Fig. 1(b) shows the transmission spectrum of the DFB grating. From tests on uniform gratings written prior to the DFB grating, the average coupling coefficient $(\kappa)$ of the DFB grating is estimated to be $\sim 39 \mathrm{~m}^{-1}$ (corresponding to an index modulation of $\sim 10^{-5}$ ). The linear tapering of the refractive index modulation profile is estimated to be $\sim 20 \%$ along the full length of the grating in the direction A to B, as shown in Fig. 1(a).

\section{Results and discussion:}

The R-DFB fiber laser was characterised by pumping it from side A (denoted AB) and side B (denoted BA) respectively, as shown in Fig. 1(a). The fiber laser was found to perform in a consistent manner using either pump direction, and in both cases it was confirmed that the laser only operates out of side A. Fig. 2(a) shows the relationship between the output power of the R-DFB and the incident pump power. The data has been corrected for the splicing loss $(0.2 \mathrm{~dB})$ between the WDM pigtail and the PS980 fiber. The threshold power was observed to be $\sim 980 \mathrm{~mW}$, which is about half the value of our previously reported results [3]. This reduction is anticipated 
because the pump source used in this work is linearly polarized in contrast to the results reported in [3] which used an un-polarised pump source. As also seen in Fig. 2(a), the slope efficiencies of R-DFB output power versus the incident pump power, with the layouts of $\mathrm{AB}$ and $\mathrm{BA}$, are identical at $7.7 \%$. The maximum output power obtained was $296 \mathrm{~mW}$ for an incident pump power of $4.9 \mathrm{~W}$. Almost $70 \%$ of the input pump power was transmitted through the system. This suggests that the remaining pump power for example could be used to amplify an additional length of fibre to form a simple MOPA configuration to boost the generated R-DFB signal as reported in [2]. The output spectra are monitored with an optical spectrum analyzer (OSA, Advantest Q8384) with RBW of $0.01 \mathrm{~nm}$, and the signal wavelength of the R-DFB is found to remain constant at $1117.71 \mathrm{~nm}$ throughout the entire power range. This suggests that the thermal effects in this laser are negligible. Fig. 2(b) shows the R-DFB output spectra at the maximum incident pump power from both sides of the grating. The signal-noise-ratio (SNR) of the R-DFB signal is seen to be $\sim 60 \mathrm{~dB}$ when measured from side A and operated in a counter pumped configuration, while there is no observed lasing signal at the signal wavelength when measured from the other side, side B. This clearly demonstrates that the R-DFB fiber laser indeed is operating with truly single-side output. The reason for such a dramatic asymmetry in the output direction ratios is not completely understood at the time of writing. A slight tapering of the refractive index profile implies that one half of the structure will have a higher reflectivity compared to the other half thereby creating an effective high reflector and output coupler, but the effects of this are more dramatic than anticipated. We are currently investigating this further and hope to report on the results of our findings at the time of the conference. Other R-DFB samples fabricated in the same manner as the device presented here all operated with a similar unidirectional output.

The linewidth performance of the R-DFB laser is tested with a delayed self-heterodyne interferometer with a $29.75 \mathrm{~km}$ delay line. The nominal resolution of this setup is $2.5 \mathrm{kHz}$ at the R-DFB signal wavelength. Fig.2(c) shows the heterodyne RF-spectra of the R-DFB fiber laser at $\sim 30 \mathrm{~mW}$ output power from side A when pumped in the $\mathrm{AB}$ and $\mathrm{BA}$ configurations, respectively. Due to the negligible thermal effects observed in the laser, only Lorentzian curves are fitted to the experimental data as seen the dashed lines in Fig. 2(c). The effects of the long coherence length of the R-DFB laser are obvious from the distinguishable sidelobes of the heterodyne spectra. The true linewidth of the R-DFB laser is therefore believed to be less than $2.5 \mathrm{kHz}$ [6]. We also examined the heterodyne RF-spectra in a wider frequency span ranging from $10 \mathrm{MHz}$ to $10 \mathrm{GHz}$ and found no evidence of any other modes oscillating in the laser. We therefore deduce that the R-DFB fiber laser indeed is a truly singlefrequency laser. The polarization extinction ratio (PER) of the R-DFB fiber laser is measured to be $>15 \mathrm{~dB}$.
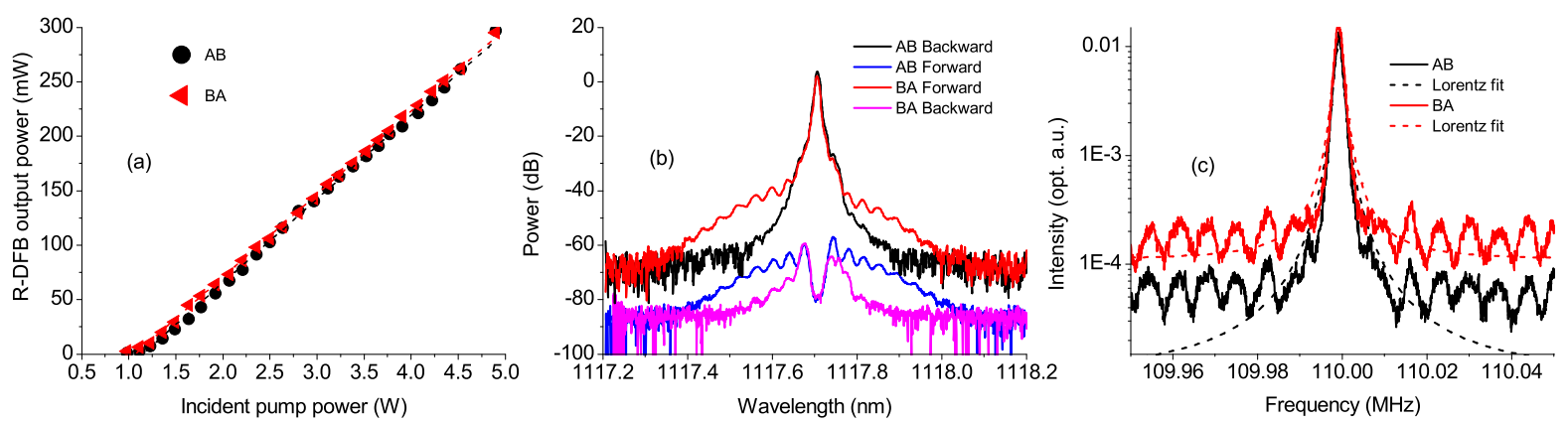

Fig. 2 (a) R-DFB output power against incident pump power; (b) Forward and backward output spectra at the maximum incident pump power; (c) Delayed self-heterodyne rf-spectra of R-DFB signal.

\section{Conclusion}

In summary, we have demonstrated a single-frequency, narrow-linewidth $(<2.5 \mathrm{kHz})$ and truly single-side output Raman DFB fiber laser at $1117.71 \mathrm{~nm}$, based on a $30 \mathrm{~cm}$ long center $\pi$ phase-shifted Bragg grating written directly in a commercially available PS980 fiber. The refractive index modulation profile is slightly tapered resulting in a dramatic one-sided output only R-DFB laser. The single-sided output performance is confirmed in both co- and counter-propagating pump configurations.

\section{References:}

[1] V. E. Perlin and H. G. Winful, "Distributed feedback fiber Raman laser," Journal of Quantum Electronics, IEEE, vol. 37, pp. 3847, 2001.

[2] P. S. Westbrook, et al., "Raman fiber distributed feedback lasers," Optics Letrers, vol. 36, pp. 2895-2897, 2011.

[3] J. Shi, et al., "High power, low threshold, Raman DFB fibre lasers," in proceedings to IQEC/CLEO Pacific Rim Sydney, Australia 28 Aug - 1 Sep 2011,postdeadline paper, 2011.

[4] M. Ibsen, et al., "Robust high power (>20 mW) all-fibre DFB lasers with unidirectional and truly single polarisation outputs," in proceedings to CLEO '99. paper CWE4, 1999.

[5] V. C. Lauridsen, et al., "Design of DFB fibre lasers," Electronics Letters, vol. 34, pp. 2028-2030, 1998.

[6] D. Derickson, "Fiber Optic Test and Measurement," Prentice Hall PTR, 1998. 